\title{
Research Paper \\ Some Clinical Features and Supportive Therapies in Covid-19 Patients Died in Hajar Hospital, Tehran, Iran
}

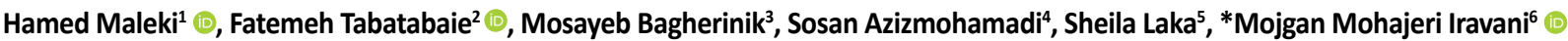

1. Department of Anesthesiology, School of Medicine, Iran University of Medical Sciences, Tehran, Iran.

2. Department of Parasitology and Mycology, School of Medicine, Iran University of Medical Sciences, Tehran, Iran.

3. Department of Crisis Management, Iran University of Medical Sciences, Tehran, Iran.

4. Department of Obstetrics and Gynecology, School of Medicine, AJA University of Medical Sciences, Tehran, Iran.

5. Department of Health, School of Public Health, Iran University of Medical Sciences, Tehran, Iran

6. Department of Anesthesiology and Intensive Care, School of Medicine, AJA University of Medical Sciences, Tehran, Iran.

\begin{tabular}{|c|c|}
\hline $\begin{array}{l}\text { Use your device toscan } \\
\text { and read the article online }\end{array}$ & n: Malek1 H Tabatabaie E B \\
\hline 口क्ष & $\begin{array}{l}\text { and Supportive Therapies in Covid-19 Patients Died in Hajar Hospital, Tehran, Iran (Persian)]. Complementary Medicine Journal. } \\
\text { 2021; 11(2):154-165. https://doi.org/10.32598/cmja.11.2.1049.2 }\end{array}$ \\
\hline 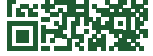 & doi)https://doi.org/10.32598/cmja.11.2.1049.2 \\
\hline
\end{tabular}

\section{(c) (i) (3)}

Article Info:

Received: 15 Feb 2021

Accepted: 28 May 2021

Available Online: 01 Jul 2021

Key words:

Covid-19, Supportive therapies, Corona-

virus

\section{ABSTRACT}

Objective Due to the spread of Coronavirus Disease 2019 (COVID-19) worldwide and the resulting mortality as well as the lack of definitive treatment for this disease, various non-invasive and invasive drugs and treatments have been used around the world for reducing the effects of this disease or accelerating the treatment process, but the positive and definite effect of none of these methods has been confirmed and they have been effective only in some cases. This study aims to investigate some clinical features and supportive therapies in deceased COVID-19 patients in Iran.

Methods This descriptive-analytical retrospective study was conducted on 100 Covid-19 patients died in Hajar Hospital in Tehran, Iran from February to September 2020. They were divided into two groups of with and without underlying diseases. After coordination and obtaining the necessary permission and informed consent, information was collected from the medical files of samples.

Results Mechanical ventilation, vasoconstrictor injection and blood products had been used as supportive therapies in deceased patients, where ventilation use $(n=62,62 \%)$ was significantly higher $(P=0.01)$ compared to vasoconstrictor injection $(n=28,28 \%)$ and blood products $(n=13,13 \%)$. Most of deceased patients had an underlying diseases (79\%). Hypertension, diabetes and heart problems were the most common underlying diseases. The disease severity, clinical manifestations, and mortality rate of deceased patients with cardiovascular diseases, diabetes and hypertension were significantly different compared to those with other underlying diseases $(P=0.01)$. The age and gender of deceased patients had no significant relationship with the use of any supportive therapies $(P \geq 0.01)$, but had a significant relationship with the underlying diseases $(P \leq 0.01)$.

Conclusion The treatment methods that are the definite needs of the patient and have more favorable effects on the recovery process have a high value in the treatment of COVID-19 patients. These patients need to use supportive therapies for purposeful and effective treatment. Mechanical ventilation is more important than injecting vasoconstrictors or using blood products.

\section{* Corresponding Author:}

Mojgan Mohajeri Iravani, PhD.

Address: Department of Anesthesiology and Intensive Care, School of Medicine, AJA University of Medical Sciences, Tehran, Iran.

Tel: +98 (21) 43822449

E-mail: mojanmoon23001@gmail.com 


\section{Extended Abstract}

\section{Introduction}

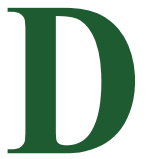

ue to the spread of COVID-19 infection worldwide and the resulting mortality, as well as the lack of a definitive treatment for it, various non-invasive and invasive drugs and treatments around the world have been used to mitigate the effects of the disease or accelerate the treatment process. However, the positive and definite effect of none of these cases has been confirmed and they have been effective only in some cases. Recent studies showed that obesity, high blood pressure, diabetes, and heart failure make it difficult to treat COVID-19. In this study, some clinical features and the need for supportive therapies in patients died due to COVID-19 were investigated to provide useful solutions for targeted recovery for patients.

\section{Materials and Methods}

This is an applied, descriptive-analytical retrospective study conducted on 100 COVID-19 patients who died in Hajar Hospital in Tehran, Iran from February to September 2020 who were selected using a census method. They were divided into two groups of with and without underlying disease. After coordination and obtaining the necessary permission and informed consent from the head of the intensive care unit, and visiting Hajar Hospital with full observance of infection control standards and full personal protection coverage, Information (Age, gender, the need for any of the supportive treatments during the hospitalization period) was collected from the medial files of samples. The patients with positive CT scan (lung scan) and PCR test who died of COVID-19 were included in the study. The criterion for excluding from the study was the death caused by other diseases. The course of the disease is classified into zero stage (with no symptoms), symptom onset stage, respiratory infection stage which is divided into two levels of moderate and severe, and finally the stage of severe and critical inflammation. The final stage is the criterion for entering supportive treatment where the patient needs special care. Criteria for entering this stage were at least one of the following cases: respiratory failure, blood oxygen saturation $\left(\mathrm{SpO}_{2}\right)<90 \%$, shock symptoms, need for mechanical ventilation or multiple organ failure. Mortality rates in the intensive care unit varies depending on the underlying disease. If necessary, according to the physician's advice and the patient's special condition, special supportive treatments are used. Statistical analysis was performed in SPSS v. 22 software using independent t-test to investigate quantitative variables with normal distribution. The significance level was set at 0.05 .

\section{Results}

According to the results, 52(52\%) of the deceased were male (mean age $=70$ years) and $48(48 \%$ ) were female (mean age $=73$ years). The deceased patients aged 36-96 years. During the hospitalization period, 62 patients $(62 \%)$ required mechanical ventilation through artificial respiration, 28(28\%) required injection of vasoconstrictor, and $13(13 \%)$ required blood product injection. The mortality rate was $25(25 \%)$ in winter, $37(37 \%)$ in spring and $38(38 \%)$ in summer. There was no significant difference

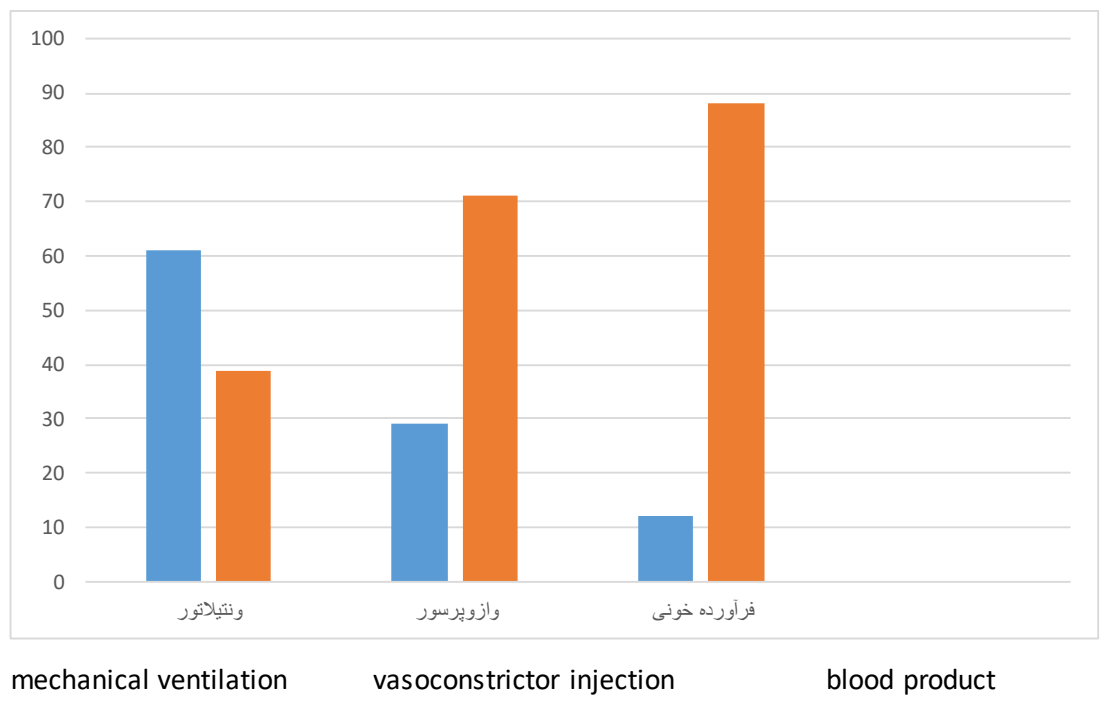

Figure 1. Data on deceased patients' use of supportive therapies

Blue color $=$ With supportive therapy; Red color $=$ Without supportive therapy 


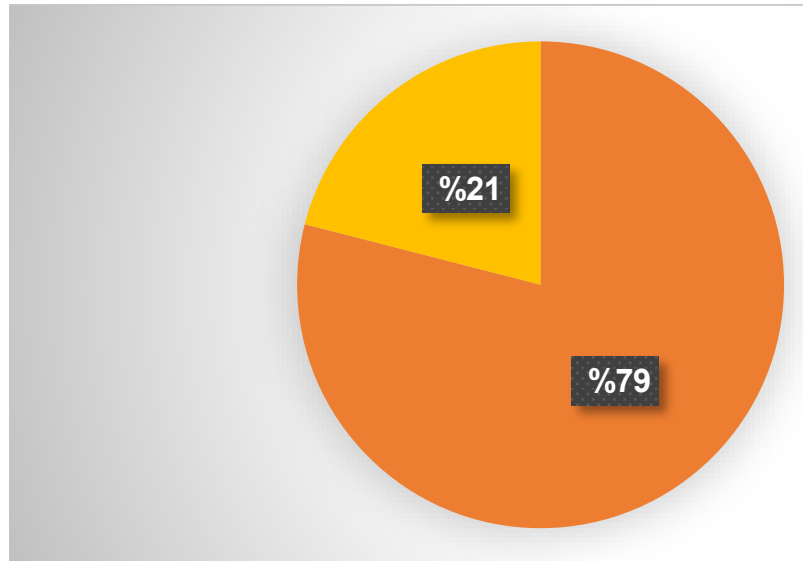

Figure 2. Percentage of deceased patients with and without underlying diseases
With underlying disease

Without underlying disease in age and gender between the two groups $(P \geq 0.05)$. The age and gender showed no significant relationship with the need for supportive treatments $(\mathrm{P} \geq 0.05)$, but had a significant relationship with underlying disease $(\mathrm{P}<0.05)$. Rapid progression of respiratory symptoms, respiratory Rate $>$ 30 per minute (with or without fever), shortness of breath, $\mathrm{SpO}_{2}<90$ in room temperature, and increased lung involvement by more than $50 \%$ were reported on CT scan of the deceased patients $(\mathrm{P}=0.01)$. These patients had entered a critical stage of disease progression. At this stage, the patient needs special care. Severe types of disease can occur at any time during the course of the disease and its occurrence does not necessarily require all previous and early stages of the disease. Patients with refractory hypoxemia, decreased level of consciousness, instability of blood flow and increased carbon monoxide in the blood with respiratory fatigue had been admitted to the intensive care unit. The cause of death was reported to be lack of contraction of the heart muscle and lack of output and blood flow in the heart or asystole.

Figure 1 shows data on deceased patients' need for supportive care. Statistical analysis of their data showed that the use of mechanical ventilation $(n=62,62 \%)$ was significantly higher than other supportive methods $(\mathrm{P}=0.01)$. It should be noted that two or more supportive therapies had not been used together. As can be seen in Figure 2, most of deceased patients had an underlying disease. Ventilators were used in 69 patients $(69 \%)$ with underlying diseases and 31 patients (31\%) without underlying diseases. The most common underlying diseases were high blood pressure, diabetes and heart problems. The severity of the disease and clinical manifestations and the mortality rate of patients with cardiovascular disease, diabetes and hypertension were significantly different than those with other underlying diseases $(\mathrm{P}=0.01)$ (Figure 3$)$.

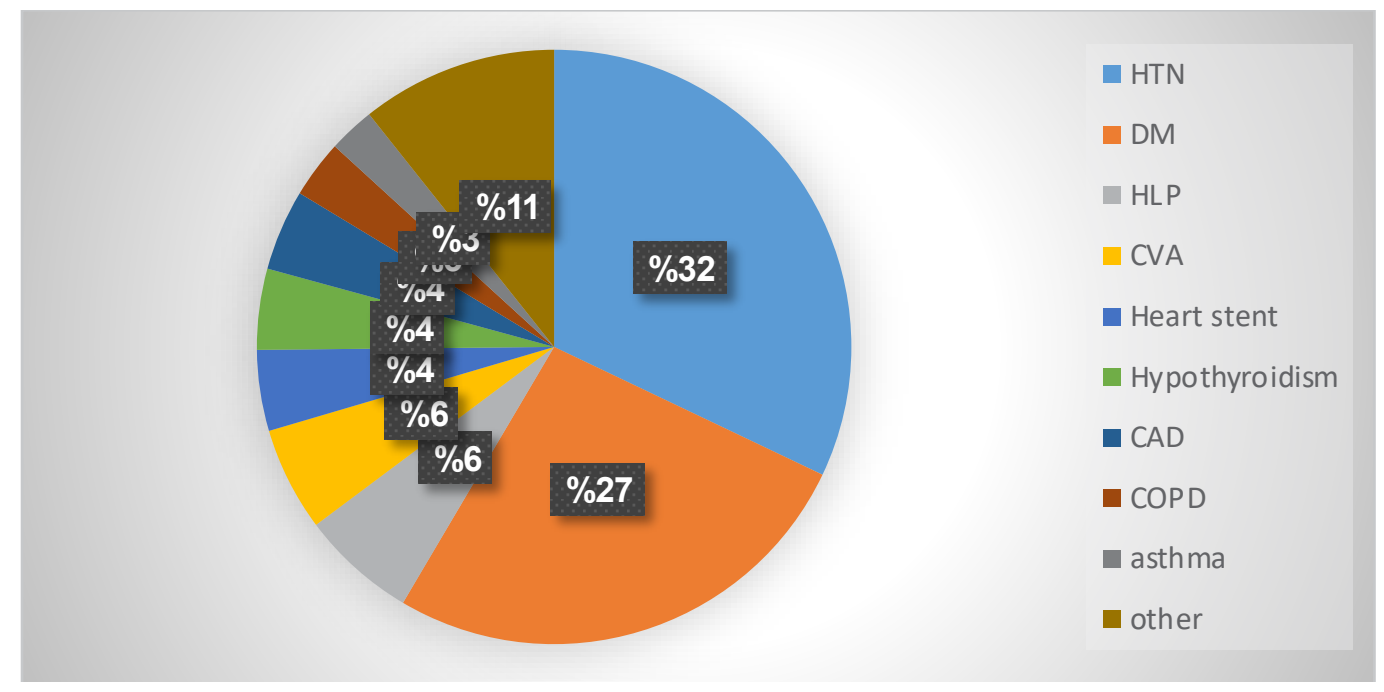

Figure 3. Percentage of underlying diseases in deceased patients 


\section{Ethical Considerations}

Compliance with ethical guidelines

This study was conducted in accordance with ethical principles in medical research on humans (Helsinki Declaration). Ethical approval was obtained from the Ethics Committee of Aja University of Medical Sciences (Code: IR.AJAUMS.REC.1399.069).

\section{Funding}

This research did not receive any grant from funding agencies in the public, commercial, or non-profit sectors.

Authors' contributions

All authors contributed equally in preparing this article

Conflicts of interest

The authors declare no conflict of interest

Acknowledgements

The authors would like to thank Iran University of Medical Sciences and AJA University of Medical Sciences for their financial support and Hajar Hospital for specialized tests 
This Page Intentionally Left Blank 


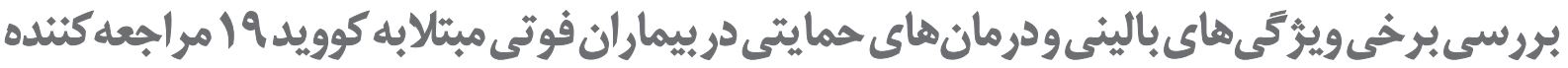

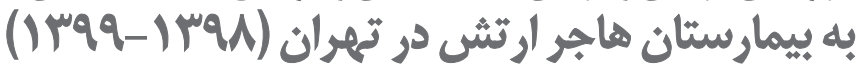

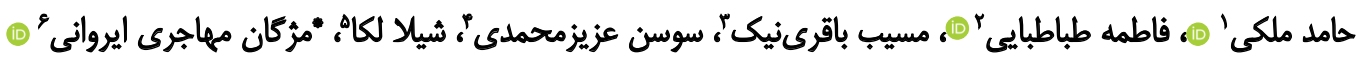

1. ا. تروه بيهوشى، دانشكده يزشكى، دانشكاه علوم يزشكى ايران، تيران، ايران.

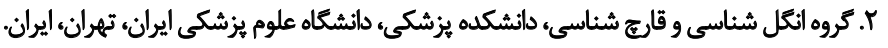

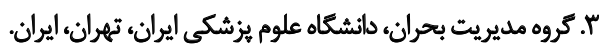

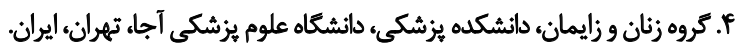

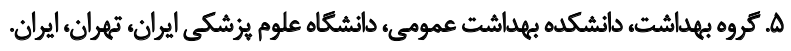

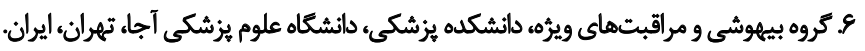

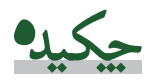

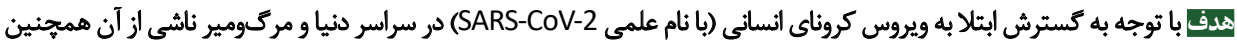

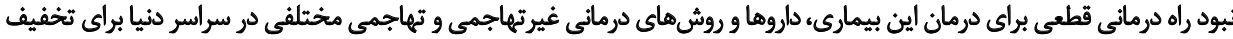

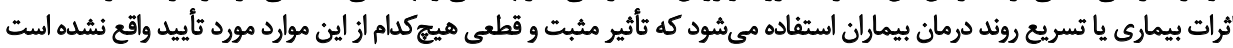

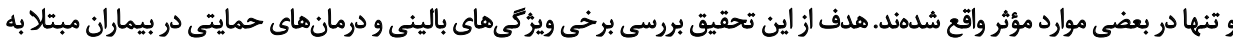

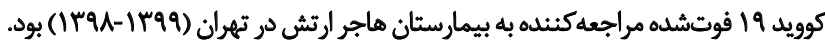

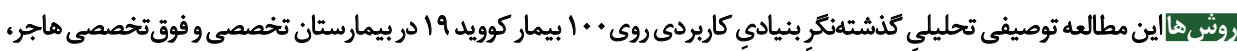

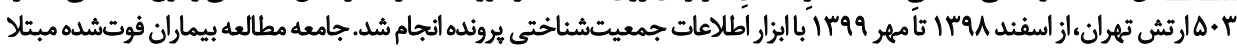

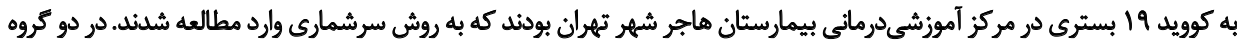

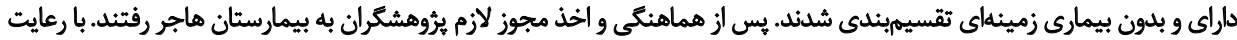

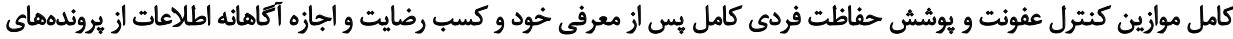

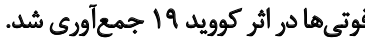

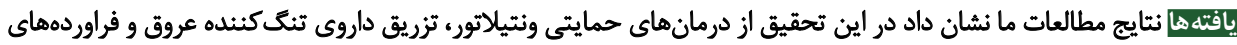

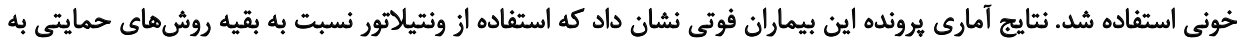

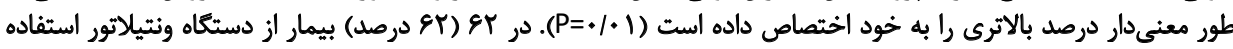

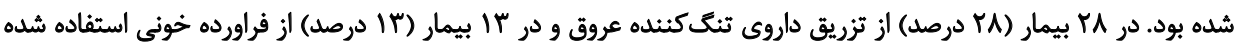

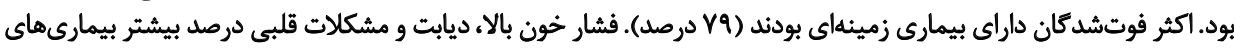

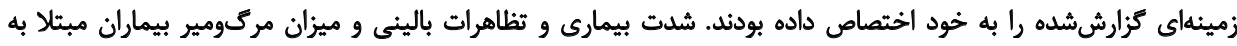

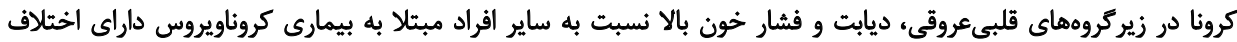

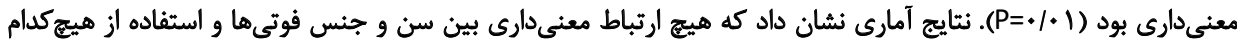

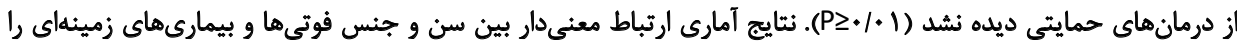

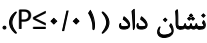

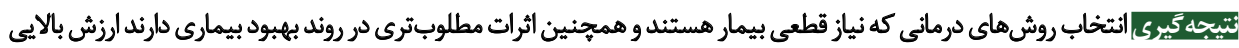

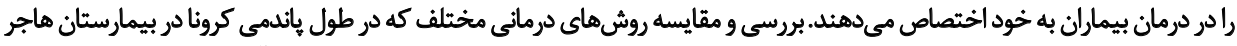

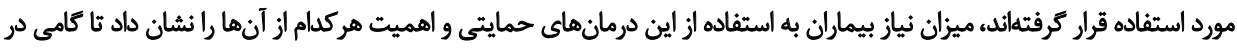

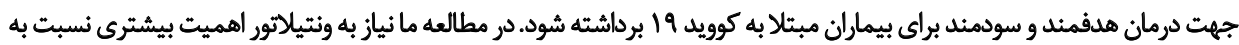

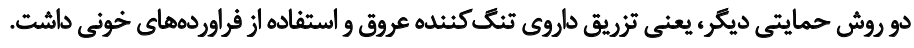

اطلاعات مقاله:

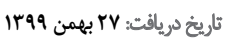

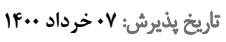

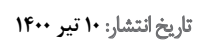

كليدواثهها: كوويد 19، درمانهاى حمايتى، بيمارستان هاجر، كروناويروس

……

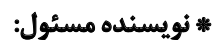

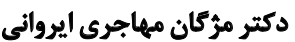

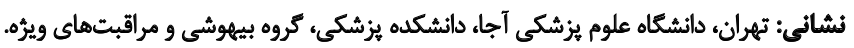

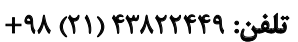

يست الكثروثيكي: mojanmoon23001@gmail.com 
به كسر اكسيرن كمتر از · ·r ميلىمتر جيوه). Y. محافظت از

مقدمه

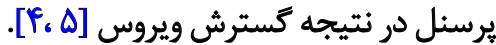

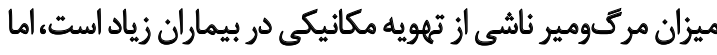

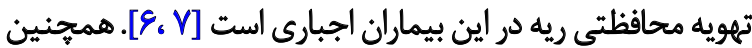

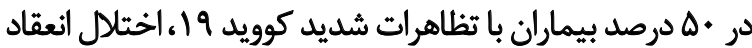

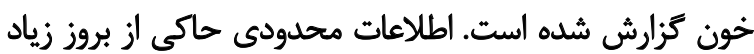

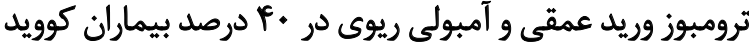

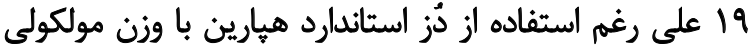

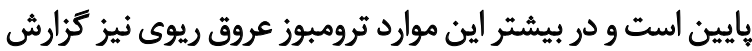

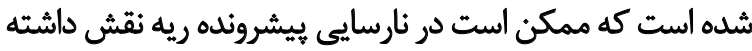

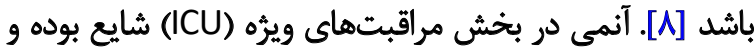

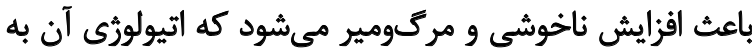

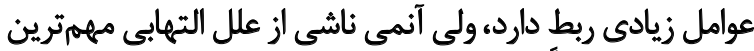

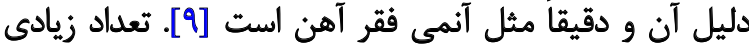

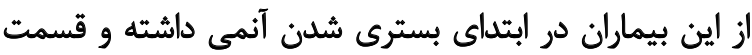

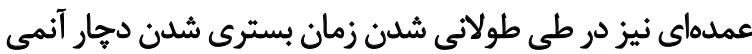

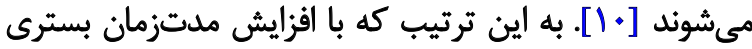

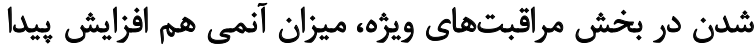

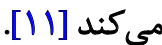

كزارشات در موارد نادرى از هنومونى كه از نوامبر ثا دسامبر

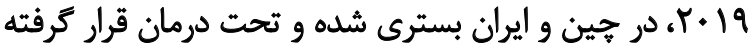

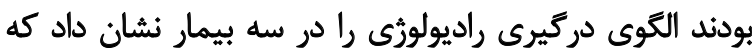
كاملاً با بيمارى

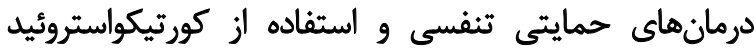

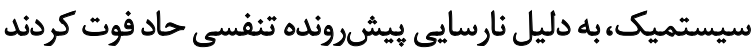

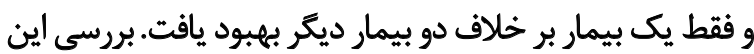

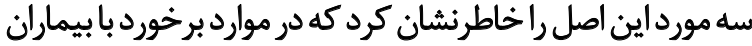

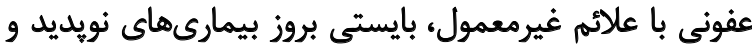

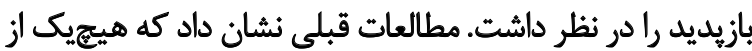

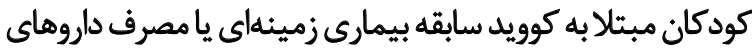

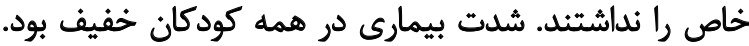

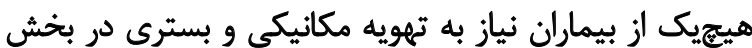

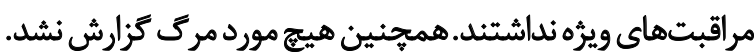

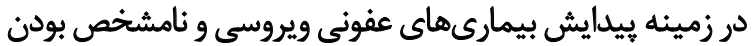

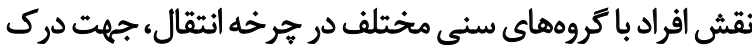

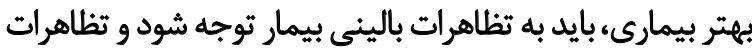

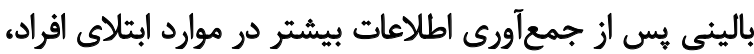

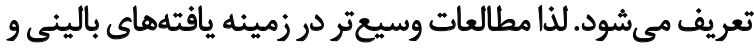

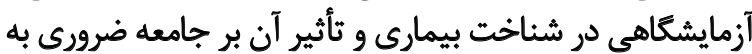

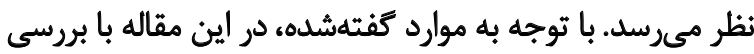

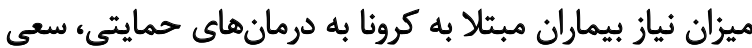

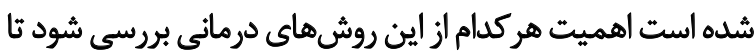

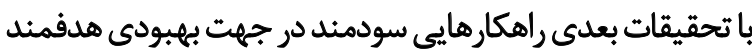

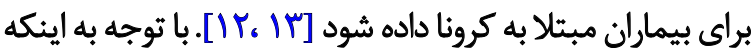

ويروس SARS-CoV-2 به عنوان يك مشكل جهانى كه از

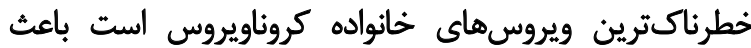

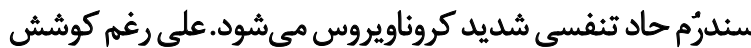

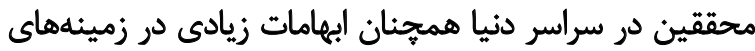

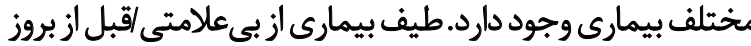

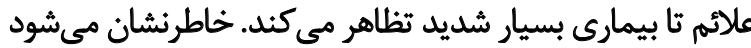

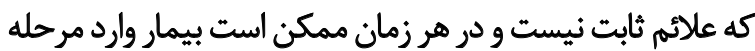

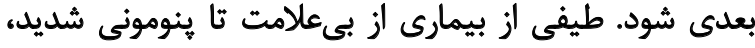

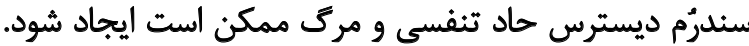

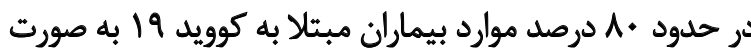

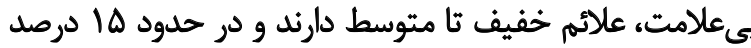

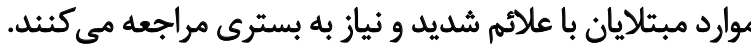

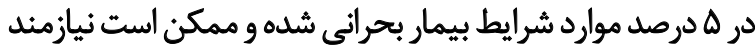

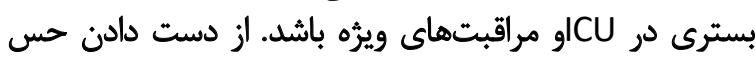

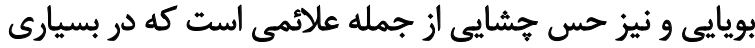

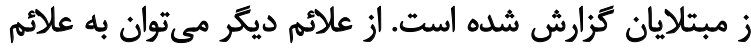

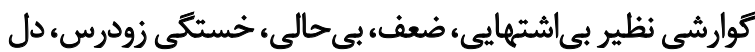

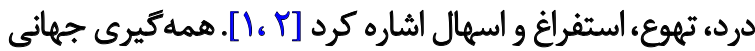

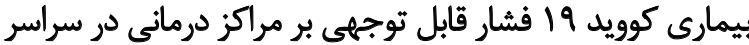

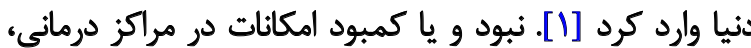

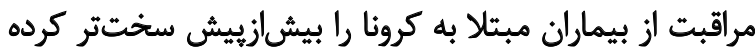

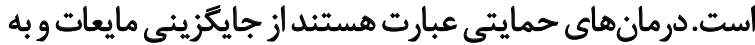

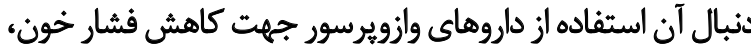

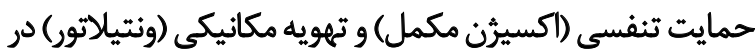

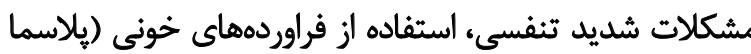

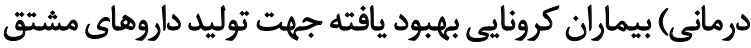

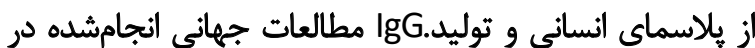

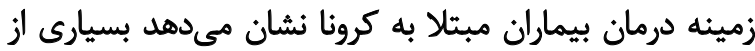

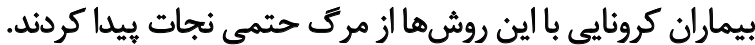

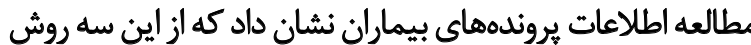

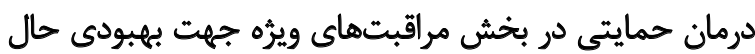

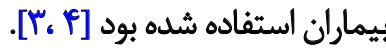

اكرجه بيشتر بيماران مبتلابه ويروس كوويد 19 انياز به بستري

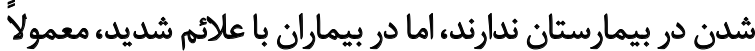

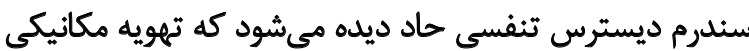

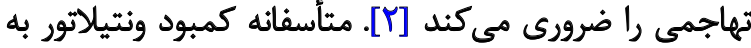

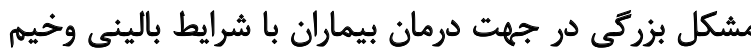

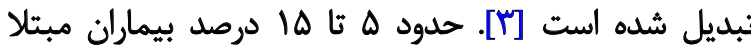

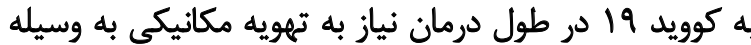

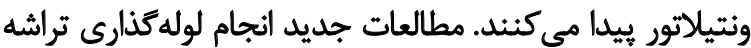

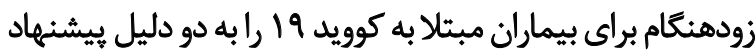

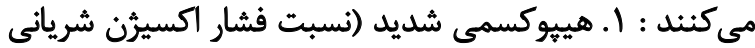




\section{يافتهها}

تحقيقات اخير نشان داده است كه جاقى، فشار خون بالا،

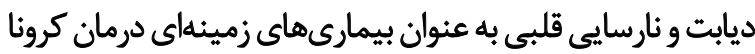

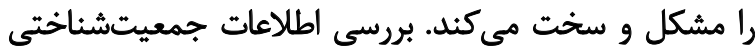

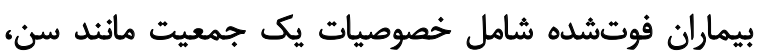

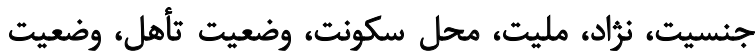

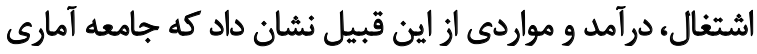

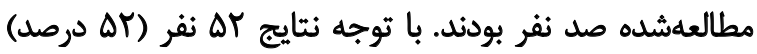

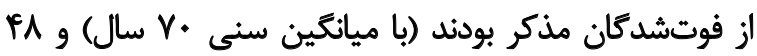

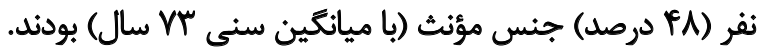

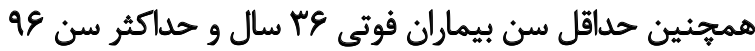

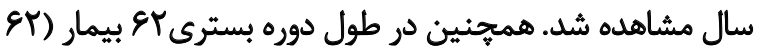

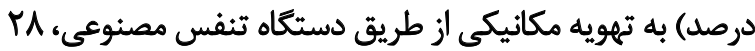

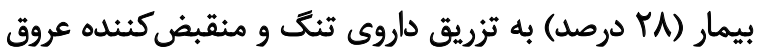

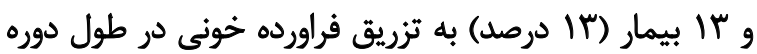

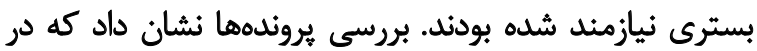

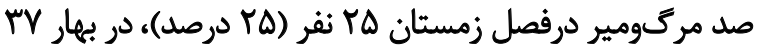

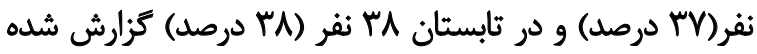

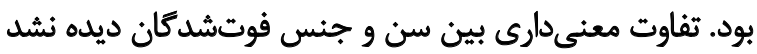

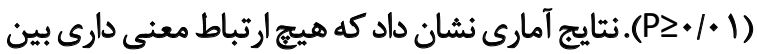

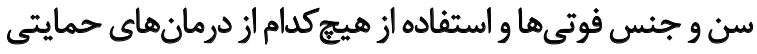

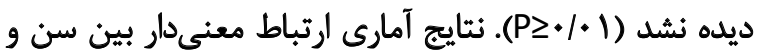

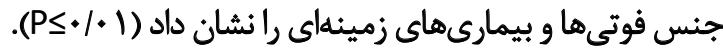

در بيماران فوتشده بيشرفت سريع علائم تنفسى، تند نفسى RR>r.

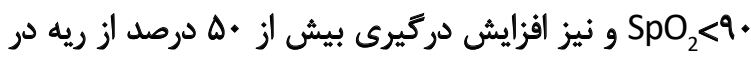
CT Scan

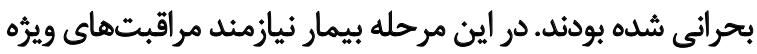

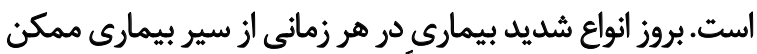

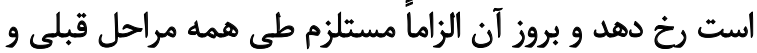

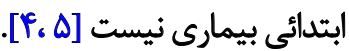

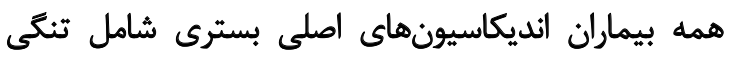

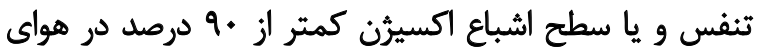

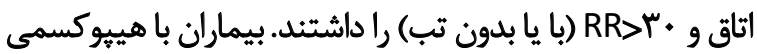

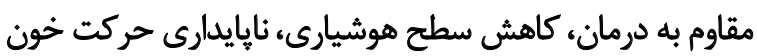

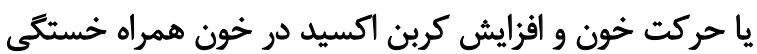

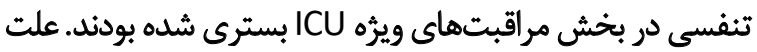

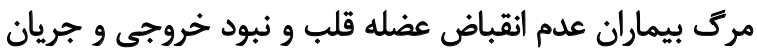

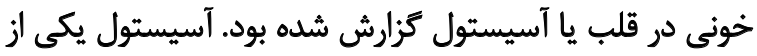

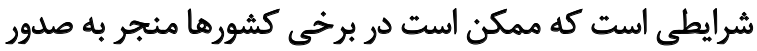

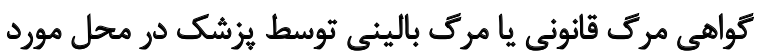

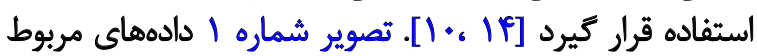

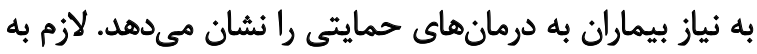

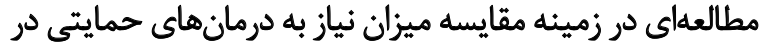

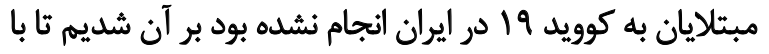

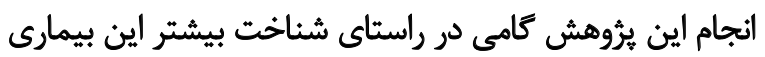

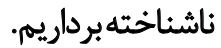

\section{مواد و روشها}

اين مطالعه، يك مطالعه تذشتهنكر بنيادى كاربردى به صورت

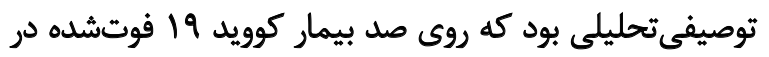

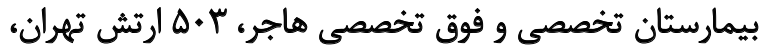

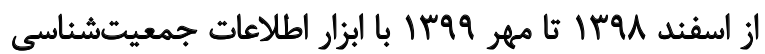

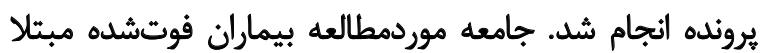

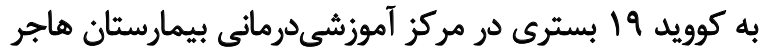

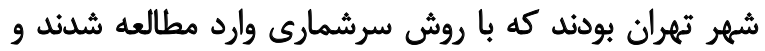

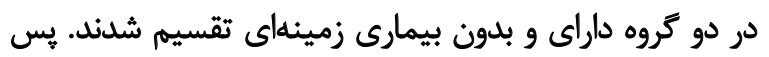

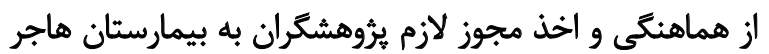

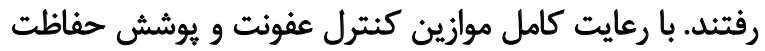

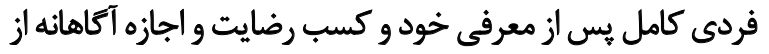

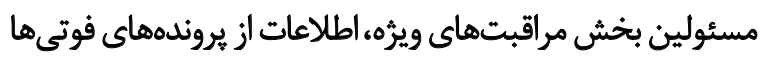

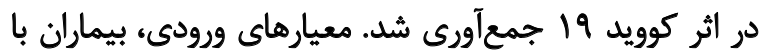

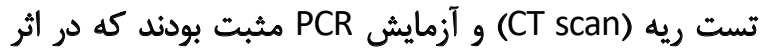

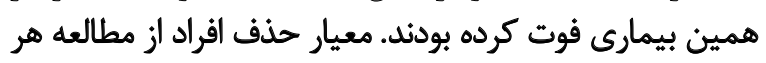

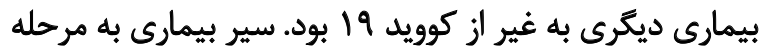

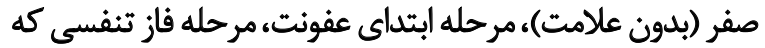

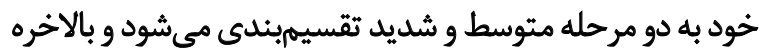

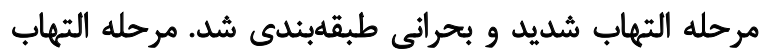

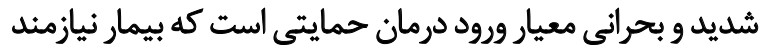

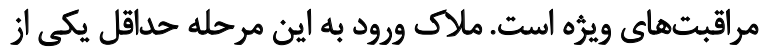

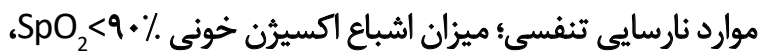

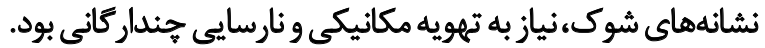
بيمارىهاى زمينهاى يرفشارى خون، ديابت كنترلنشدها،

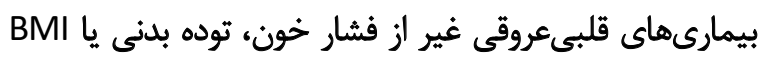

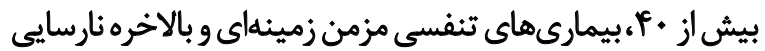

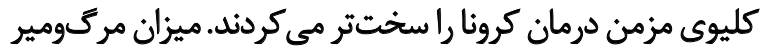

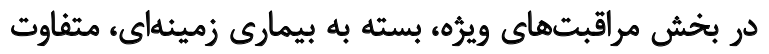

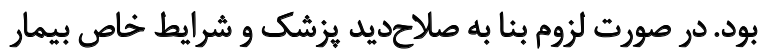

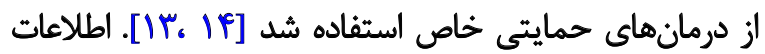

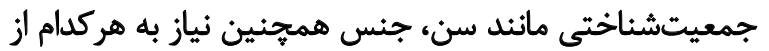

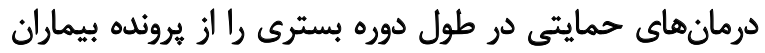

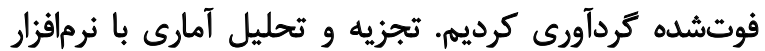

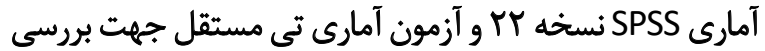

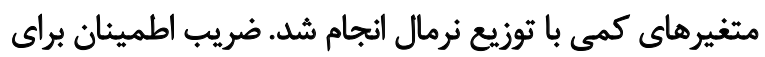

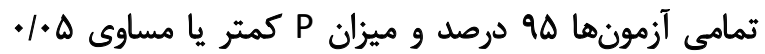
معنى دار در نظر كرفته شد. 


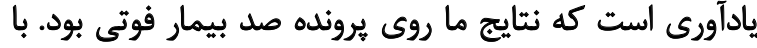

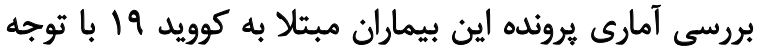
بيمارى مسرى كوويد 9 الز بيمارىهاى مشترك بين انسان و

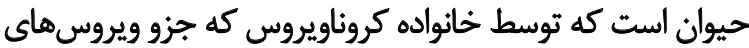
RNA

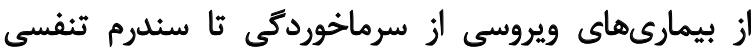

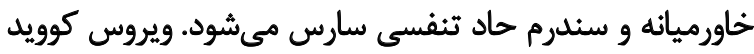

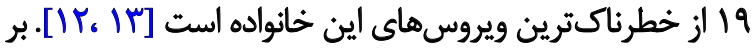

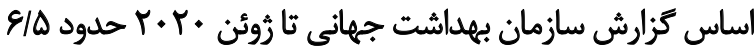

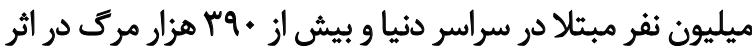

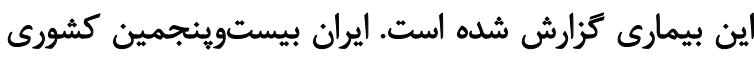

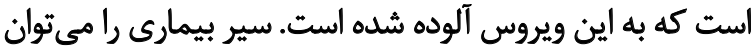

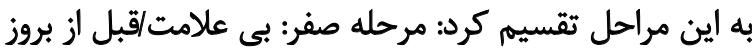

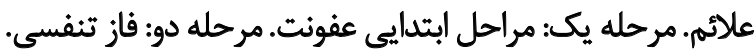

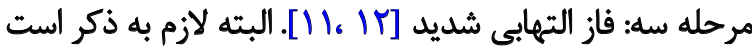

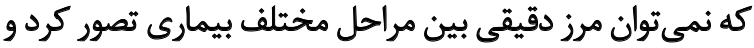

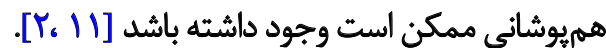

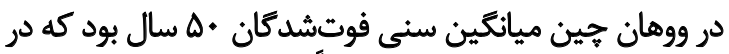

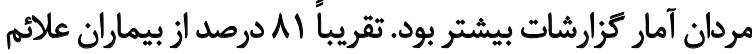

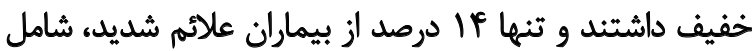

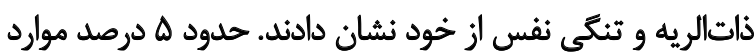

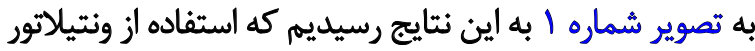

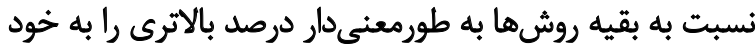

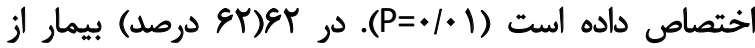

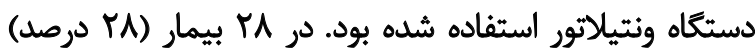

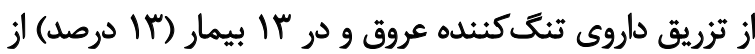

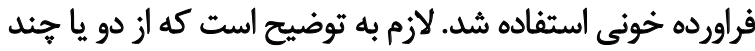

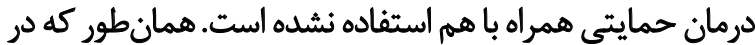

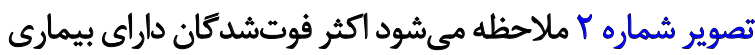

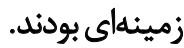

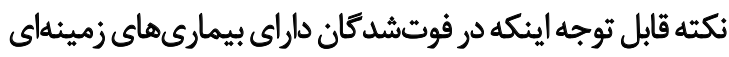

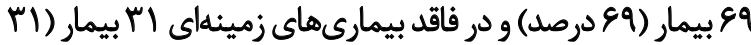

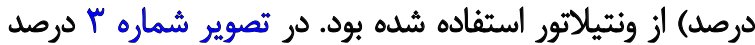

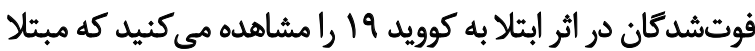

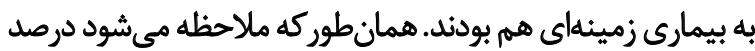

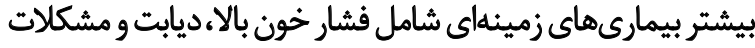

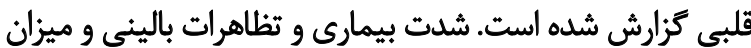

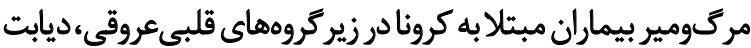

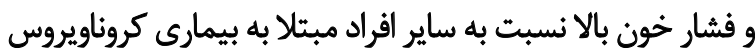

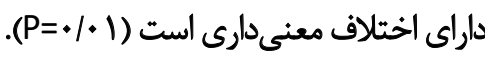

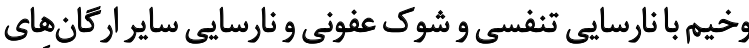
بدن مواجه شدند. تب و سرفه از رايجترين علائم مخصوصاً در

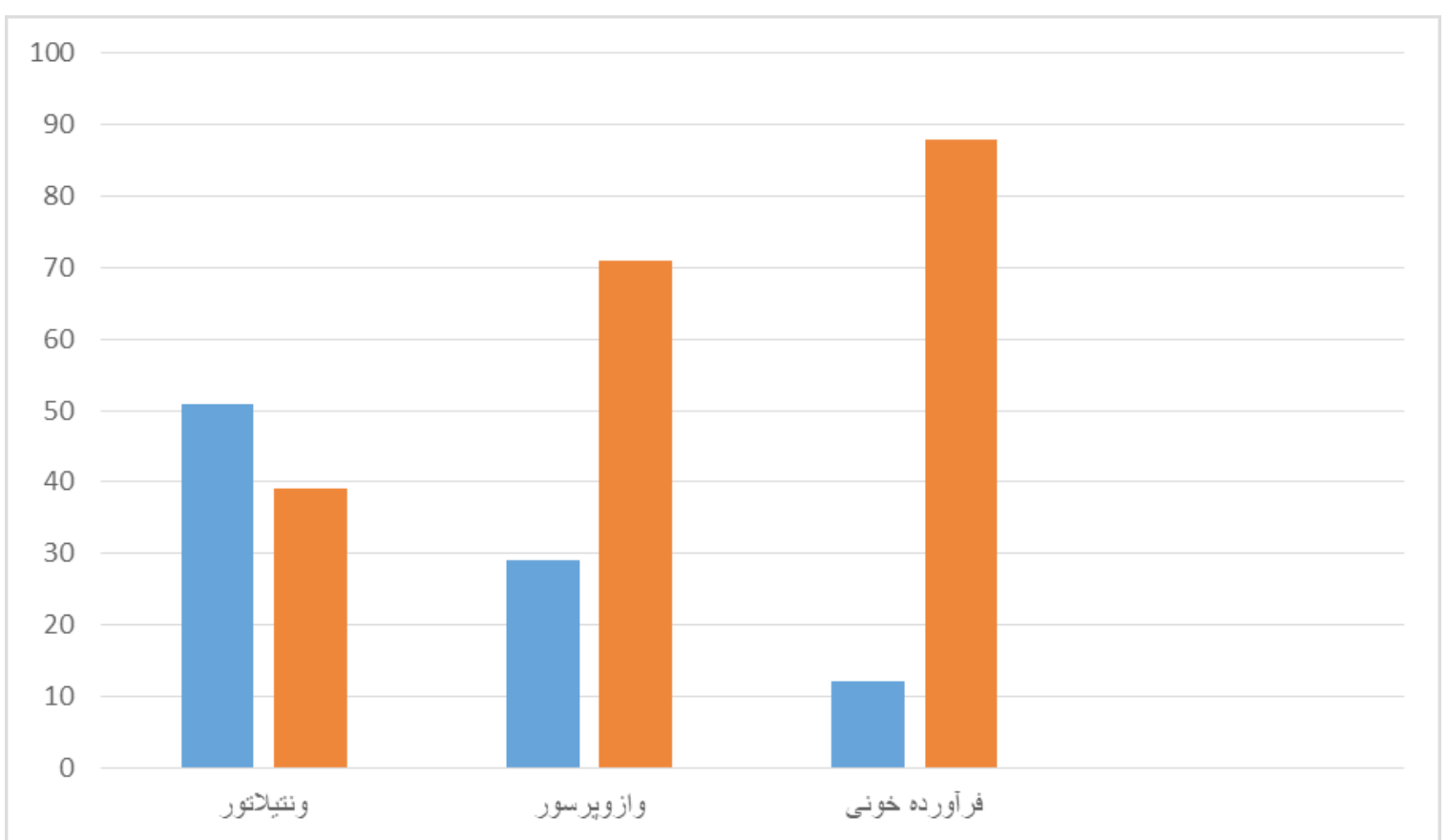




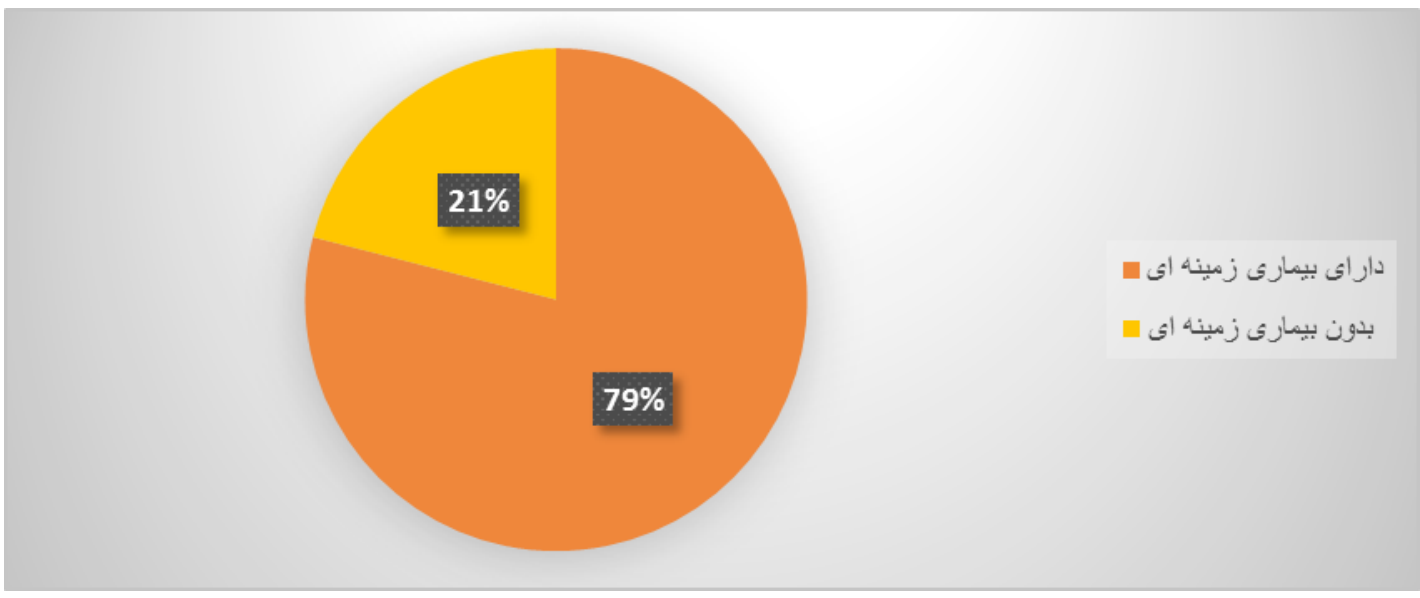

توجه به نشائها و علائم بالينى بيمارانى كه فوت كردماند نشان

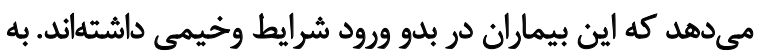

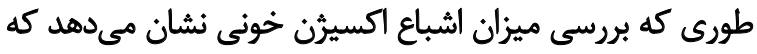
بيشتر اين بيماران Spo

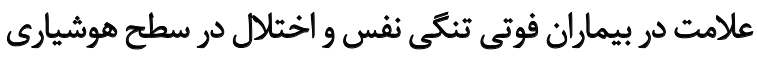

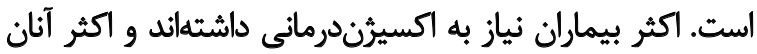

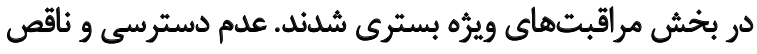

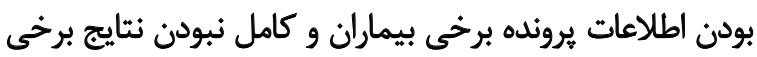

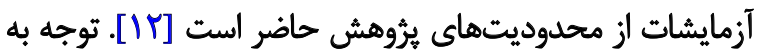

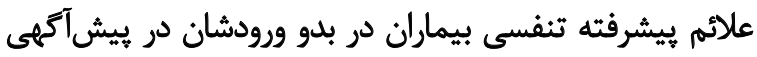

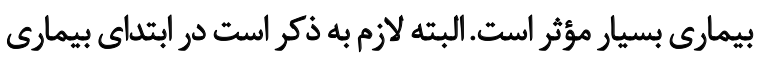

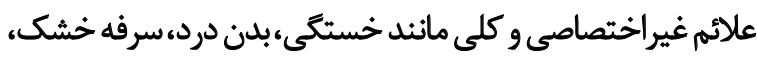

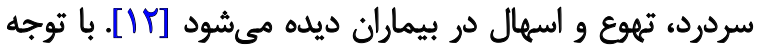

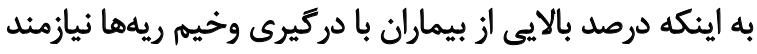

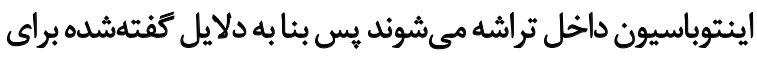

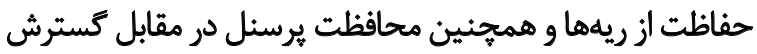

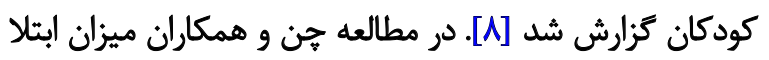

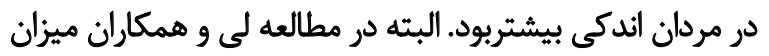

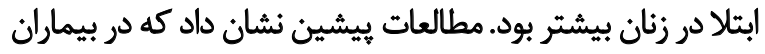

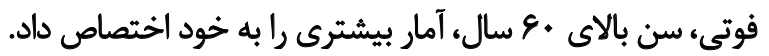

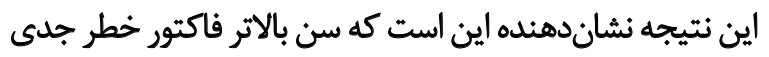

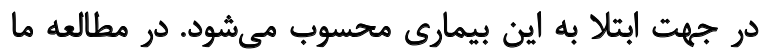

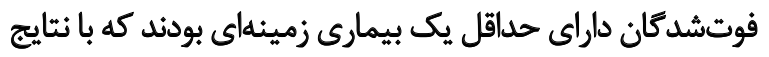

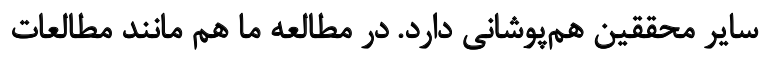

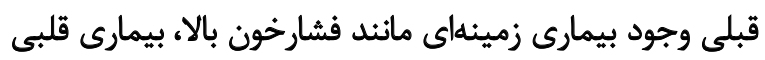

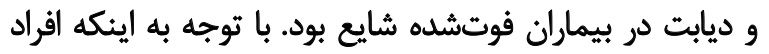

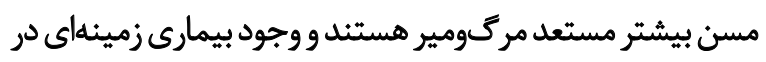

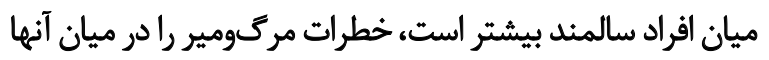

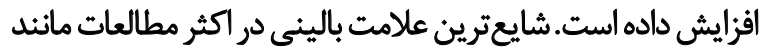

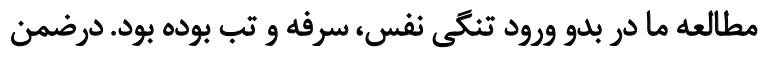

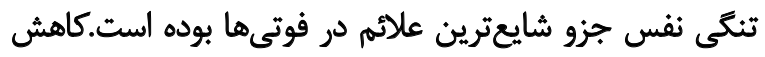

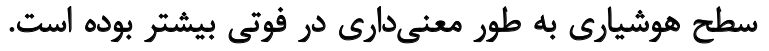

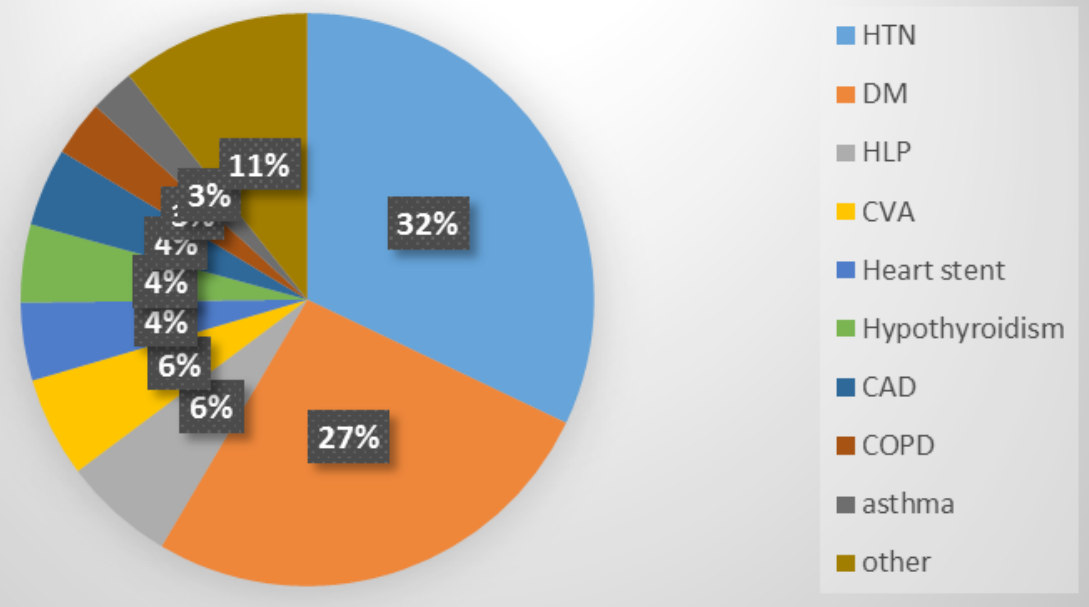




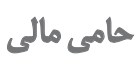

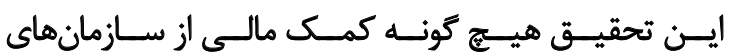

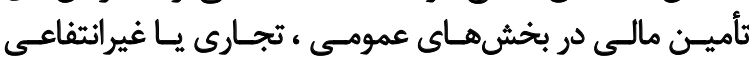

$$
\text { دريافت نكـرد. }
$$

$$
\text { مشاركت نويسندكًان }
$$

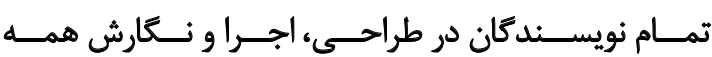

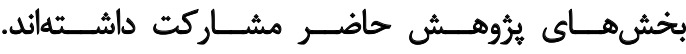

$$
\text { تعارض مثاقع }
$$

نويسندكان تصريح مى كنيد كه هيجگُونه تضاد منافعى وجود

$$
\text { تشكر وقدرداني }
$$

نويسندكان اين مقاله از دانشكاه علوميرُشكى ايران و دانشكاه

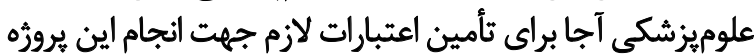

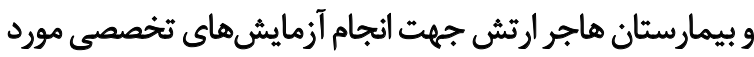

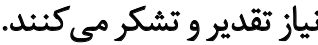

ويروس، بهتر است يروتكلهايى براى اينتوباسيون زودهنكام

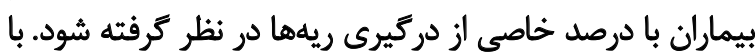

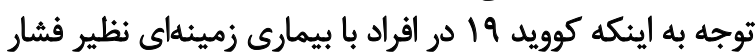

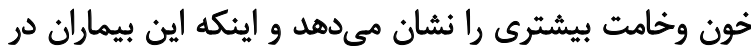

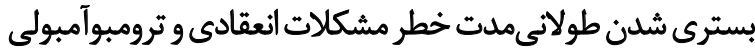
بيشترى بيدا مى كنند، اين مسئله نيز اهميث استفادها از داروهاى

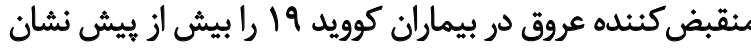

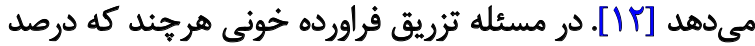

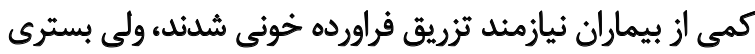

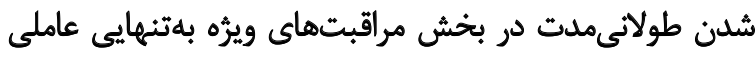

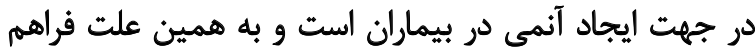

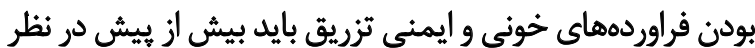

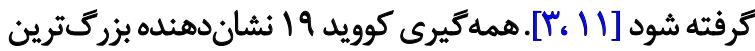

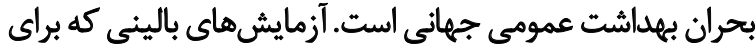

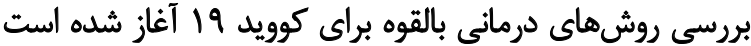

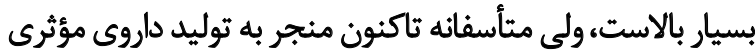

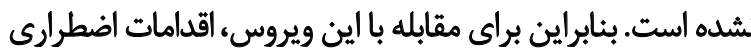

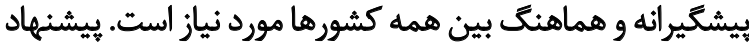

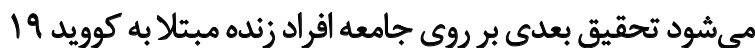
و مقايسهاى بين نتايج دو كروه انجام شود.

\section{تئيجليكيرى}

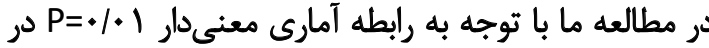

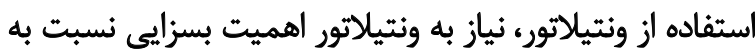

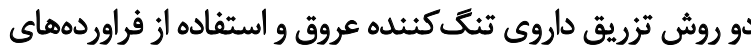

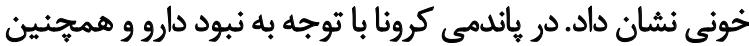

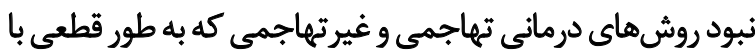

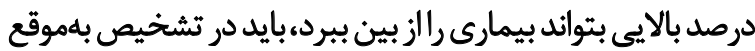

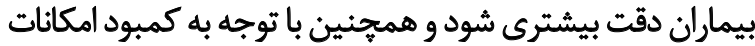

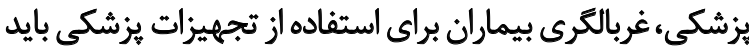

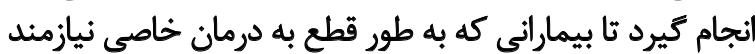

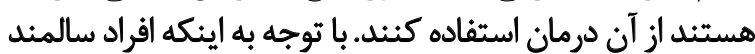

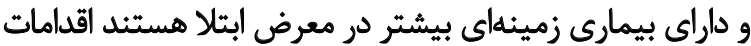
بيشغيرانه و احتياطى براى آنان لازم وضرورى است.

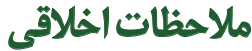

بيروى از اصول اخلاق يُوهش كد اخلاق اين يزؤه

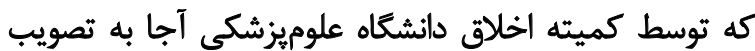

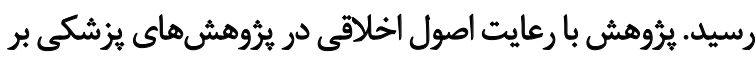
روى انسان (بيانيه هلسينكى) و بر اساس كد اخلاق انجام شده بره 


\section{References}

[1] Emanuel EJ, Persad G, Upshur R, Thome P, Parker M, Glickman A, et al. Fair allocation of scarce medical resources in the time of Covid-19. The New England Journal of Medicine. 2020; 382:2049-2055. [DOI:10.1056/ NEJMsb2005114] [PMID]

[2] Arentz M, Yim E, Klaff L, Lokhandwala S, Riedo FX, Chong M, et al. Characteristics and outcomes of 21 critically ill patients with COVID-19 in Washington State. JAMA. 2020; 323(16):1612-4. [DOI:10.1001/ jama.2020.4326] [PMID] [PMCID]

[3] Biddison LD, Berkowitz KA, Courtney B, De Jong CM, Devereaux AV, Kissoon $\mathrm{N}$, et al. Ethical considerations: care of the critically ill and injured during pandemics and disasters: CHEST consensus statement. Chest. 2014; 146(4):e145S-55S. [DOI:10.1378/chest.14-0742] [PMID]

[4] Phua J, Weng L, Ling L, Egi M, Lim CM, Divatia JV, et al. Intensive care management of Coronavirus Disease 2019 (COVID-19): Challenges and recommendations. The Lancet Respiratory Medicine. 2020; 8(5):50617. [DOI:10.1016/S2213-2600(20)30161-2]

[5] Alhazzani W, Møller MH, Arabi YM, Loeb M, Gong MN, Fan E, et al. Surviving sepsis campaign: Guidelines on the management of critically ill adults with Coronavirus Disease 2019 (COVID-19). Intensive Care Medicine. 2020; 46(5):854-87. [DOI:10.1007/s00134-020-06022-5] [PMID] [PMCID]

[6] Gattinoni L, Coppola S, Cressoni M, Busana M, Rossi S, Chiumello D. COVID-19 does not lead to a "typical" acute respiratory distress syndrome. American Journal of Respiratory and Critical Care Medicine. 2020; 201(10):1299-300. [DOI:10.1164/rccm.202003-0817LE] [PMID] [PMCID]

[7] Gattinoni L, Chiumello D, Caironi P, Busana M, Romitti F, Brazzi L, Camporota L. COVID-19 pneumonia: different respiratory treatments for different phenotypes? Intensive Care Medicine. 2020; 46:1099-102. [DOI:10.1007/s00134-020-06033-2] [PMID] [PMCID]

[8] Yang X, Yu Y, Xu J, Shu H, Liu H, Wu Y, et al. Clinical course and outcomes of critically ill patients with SARS-CoV-2 pneumonia in Wuhan, China: A single-centered, retrospective, observational study. The Lancet Respiratory Medicine. 2020; 8(5):475-81. [DOI:10.1016/S2213-2600(20)30079-5]

[9] Prakash D. Anemia in the ICU: Anemia of chronic disease versus anemia of acute illness. Critical Care Clinics. 2012; 28(3):333-43. [DOI:10.1016/j. ccc.2012.04.012] [PMID]

[10] Vincent JL, Abraham E, Kochanek P, Moore FA, Fink MP. Textbook of critical care e-book. Amsterdam: Elsevier Health Sciences; 2011. https:// books.google.com/books?hl=en\&lr=\&id=uAl68tCzm5IC\&oi=fnd\&pg=

[11] Rodriguez RM, Corwin HL, Gettinger A, Corwin MJ, Gubler D, Pearl RG. Nutritional deficiencies and blunted erythropoietin response as causes of the anemia of critical illness. Journal of Critical Care. 2001, 16:3641. [DOI:10.1053/jcrc.2001.21795] [PMID]

[12] Talebi S, Nematshahi M, Tajabadi A, Khosrogerdi A. [Comparison of clinical and epidemiological characteristics of deceased and recovered patients with COVID-19 in Sabzevar, Iran (Persian)]. Journal Military Medicine. 2020; 22(6):509-16. http://militarymedj.ir/article-1-2580-en. html

[13] Lal Kheirkhah E, Haddadi M, Ardakani M, Adel Barkhordar A, Taraz Z, Yazdi S. [Clinical demonstrations, laboratory and radiological findings of pediatric patients with COVID-19 in esfarayen-a case series (Persian)]. Journal of Sabzevar University of Medical Sciences. 2021; 27(6):796-800. http://jsums.sinaweb.net/article_1342_0.html?lang=en
[14] Forbes $P$, Finch $A$. Redeployed staff and better teamwork: How COVID-19 has transformed nursing. 2020; 27(4):14-17. [DOI:10.7748/ nm.27.4.14.s11] 